\title{
Current Status and Future Needs of Pediatric Nephrology Position Paper from American Society of Pediatric Nephrology
}

\section{SPECIFIC RECOMMENDATIONS}

1. Increased support for training pediatricians to become nephrologists and basic and clinical investigators in the area of pediatric nephrology.

2. Specific grant programs to support relevant basic and clinical research for pediatric nephrology.

a. Four broad categories of research activities of high relevance to central issues in pediatric nephrology have been identified.

b. Key personnel at various funding agencies and especially at the National Institules of Health (NIH) need to be made aware of research needs in these areas for the potential development of Request for Applications (RFA).

c. Appropriate mechanisms for the review of proposals directed toward these research questions are warranted.

1. Increase by $10 \%$ the number of pediatric nephrologists on study sections and initial review groups to which such proposals would be submitted.

2. Consider an Ad Hoc Study Section on an annual basis composed of pediatric nephrologists sitting on other Study Sections or who have served previously on NIH Study Sections.

3. Constitute a Special Study Section dedicated to reviewing applications in response to RFA directed toward these questions.

3. Expanded support for multicenter clinical studies on the etiology and therapy of kidney disease in children.

4. Creation of two pediatric nephrology centers with a multiuniversity location.

\section{INTRODUCTION}

After considerable and deliberate consultation, the American Society of Pediatric Nephrology (ASPN) has developed this position paper concerning the special needs of the field of pediatric nephrology. The intent of this paper is to stimulate support for the program needs of the discipline of pediatric nephrology. This report will address several key factors which threaten the long-term productivity, high level of scholarship, and scientific inquiry in pediatric nephrology.

\section{BACKGROUND}

The formative years of the discipline of pediatric nephrology were between the end of the second world war and the mid1960 s, beginning with the finding of remarkable differences in renal function between infants and adults. The driving force for the evolution of the discipline of pediatric nephrology was the simultaneous recognition that antibiotics and glucocorticoids could be used to treat the nephrotic syndrome, the application of the technique of renal biopsy to study of childhood glomerular

Correspondence Russell W. Chesney, M.D., Chairman, Department of Pediatrics, Le Bonheur Children's Medical Center, 848 Adams Avenue, Memphis, TN 38103 . disease, and a clear recognition of the distinct differences in natural history and treatment of children with renal disease as compared to adults. As pediatric nephrologists addressed the broad spectrum of renal disease, the unique impact of chronic renal insufficiency on children became apparent, and the challenge to adapt techniques of dialysis and transplantation for the care of uremic infants, children, and adolescents was mandated. As a focus for scientific activities, the American Society of Pediatric Nephrology was founded 20 years ago, with Walter Heymann as its first president. This organization has served to disseminate information to physicians and life scientists interested in this new discipline and to stimulate scientific investigations. The Society has held an annual scientific meeting since and currently has a membership of 350 physicians.

Accomplishments over the past two decades include the expansion of our knowledge of renal physiology to include the fetus and the newborn, a far greater understanding of immunopathogenesis and classification of glomerular disorders, implementation of sophisticated therapy (including hemodialysis, peritoneal dialysis, hemofiltration, plasmapheresis, and hemoperfusion) for children almost regardless of size, the widespread use of renal transplantation to infants, children, and adolescents, and development of strategies aimed at improving growth, nutrition, and bone disease in children with moderate or severe renal insufficiency. Paramount to the expansion of knowledge was the appearance of several multicenter groups dedicated to the study of childhood renal disease using the power of greater patient number and more physician investigator input. A section of pediatric nephrology is considered an integral part of a contemporary department of pediatrics and has a mandated role in the education of pediatric residents. The American Board of Pediatrics requires that general pediatric training programs include the resource of pediatric nephrology.

Currently, 30 training programs have been certified by the Residency Review Committee of the Accreditation Council for Graduate Medical Education (ACGME) to provide special training in pediatric nephrology. The certification process included a careful scrutiny of both clinical and research activities. This included evaluation of the adequacy of clinical material, an organized and dedicated end stage renal disease program for children and rigorous training in scientific methodology and research. In accordance with the regulations and requirements of the sub-board of Pediatric Nephrology of the American Board of Pediatrics, each trainee must undergo at least 3 years of postresidency fellowship training and is tested for competency and knowledge of general nephrology, immunology, renal physiology, end stage renal disease care, transplantation, and genitourinary tract abnormalities, as well as research methods.

At present, pediatric nephrologists are involved in clinical and basic research, and utilize both classical and modern techniques for investigating the spectrum from whole organ to cellular and molecular biology. Investigators use techniques including cell and organ culture, cell and molecular biology, micropuncture, microperfusion, isolated membrane and separated tubule segment preparations and hemodynamic determinations. Other in- 
vestigators are involved in the study of renal metabolic function, growth factors, receptor and signal transduction, hypertrophy and a panoply of immunology methods including cellular immunobiology, immunopathology, and immunogenetics, particularly of subcellular components of the glomerular basement membrane and of tubular epithelium. These investigations ask questions regarding childhood renal disorders and the developing fetal, neonatal and maturing kidney, stressing the unique function of the fetal and developing kidney to meet the growth needs of the organism and the congenital and hereditary renal disorders of childhood. Other investigators examine clinical physiologic problems, electrolyte disorders, hypertension, and urinary tract infections. The results of these research endeavors is the establishment of an ever-expanding data base for the discipline of pediatric nephrology. In addition, pediatric nephrologists have made and continue to make contributions to the broader science of nephrology. In the recognition of their accomplishments, there has been a recent increase in the number of NIH-funded research proposals in pediatric nephrology from 12 in 1982 to 32 in 1986.

Fewer medical graduates in the United States are selecting training programs for primary care specialties in both internal medicine and pediatrics, and of those who do, the percentage electing subspecialty training in nephrology each year has declined. For example, there are 2020 PL-1 positions in general pediatrics proposed for July 1988, but it is expected that only $85 \%$ of these will be filled. Of those who complete pediatric residencies, $60 \%$ will practice general pediatrics, and $40 \%$ will elect further training. According to the American Board of Pediatrics, $0.6 \%$ of potential candidates for the examination between 1981 and 1984 had chosen a career in nephrology. Of the 1090 medical school graduates in the United States who completed pediatric training in 1987 and were candidates for the written examination, $9(0.8 \%)$ were planning further training in nephrology as compared to $2.5 \%$ to $5 \%$ of candidates planning careers in other major pediatric subspecialties. Only $0.3 \%$ of candidates for the oral examination had actually embarked on nephrology training. The trend in nephrology training among internists is similar-a $20 \%$ decrease since 1980 . Twenty years ago, nephrologists in internal medicine provided care for older children and adolescents with renal disease when no pediatric nephrologist was accessible. However, treatment of chronic renal insufficiency in neonates, infants, and young children appropriately has not been assumed by internists in most communities, but the shortage of pediatric nephrologists has caused some pediatric programs at major institutions in the United States to recruit nephrologists from internal medicine to assume full-time responsibilities for pediatric nephrology.

Among the reasons given by pediatricians for not selecting nephrology for subspecialty training include financial debt incurred during college and medical school and the inability/ unwillingness to postpone repayment of loans for an additional 3 years during training. An exclusive private practice of pediatric nephrology, unlike most subspecialties, is not feasible even in metropolitan areas. Job opportunities exist, therefore, only in academic settings, usually located in urban areas. Furthermore, the choice of lifestyles is not conducive to a career in pediatric nephrology. Reasons given by pediatric nephrologists for leaving the subspecialty for administrative posts, private practice of general pediatrics, or industry, is summed up as poor job satisfaction. For instance, patient care becomes burdensome, remains constant for inpatients and outpatients, and confines the nephrologist-often the only such specialist-to a large geographic region, and its practicing physicians, with whom the pediatric nephrologist must coordinate his/her own patients' management across hundreds of miles. Such conditions are suboptimal; often the patient cannot be examined and information must be given by telephone; medical jurisprudence does not support this kind of patient management. This kind of commitment required of the pediatric nephrologist disrupts family life, vacation plans, and attendance at scientific meetings. Moreover, the ability to compete for and maintain extramural funding for research is impaired by the clinical workload. As long as the measure of academic productivity remains the number of publications in peer-reviewed journals, the prospect for a pediatric nephrologist with major clinical responsibilities being promoted and achieving tenure is modest. Finally, but not unimportantly, the salary of an experienced pediatric nephrologist working 60 hours a week often only equals or is less than that of a general pediatrician employed by an HMO providing better fringe benefits for 40 hours a week and less responsibility.

This position paper reflects the opinions and deliberations of leaders in the field of pediatric nephrology concerning needs in the 1990s. It was written after considerable consultation with the individuals listed at the end of this document and was finally put together by a writing subcommittee of the larger group.

\section{MANPOWER NEEDS}

In a recent (1987) survey conducted by the American Society of Pediatric Nephrology, $95 \%$ of 126 chair program directors of medical school-affiliated pediatric training programs in the United States (69\% of 16 programs in Canada) completed and returned the questionnaire. The results confirmed that a serious manpower shortage in pediatric nephrology extant already will become even more critical over the next 5 years. This finding is in marked contrast to situations in Europe, Australia, and Japan, where experienced pediatric nephrologists cannot find permanent positions.

According to the survey, 221 academic positions in pediatric nephrology are filled currently in the United States (16 in Canada), i.e. approximately 1 pediatric nephrologist per million population or 1 pediatric nephrologist for every 200,000 children. By comparison, there are an estimated 3011 nephrologists in internal medicine-14 adult nephrologists per million population or 1 nephrologist for every 60,000 adults. Pediatric nephrologists work in 40 of the 50 states, as well as in the District of Columbia and Puerto Rico; 10 states (AK, ID, KS, ME, MS, MT, NM, ND, WV, WY) have no resident pediatric nephrologist, 11 states (AZ, DE, HI, NE, NH, NV, OR, RI, SD, VT, WA) have 1 pediatric nephrologist and 7 states (AL, AR, CO, GA, IN, OK, SC) and Puerto Rico have 2 pediatric nephrologists working together in the same major city. Of the 240 pediatric training programs in the United States now required to have pediatric nephrology expertise for patient care and teaching housestaff to maintain accreditation, $56 \%$ have no consult in pediatric nephrology, $17 \%$ have $1,14 \%$ have $2,6 \%$ have 3 , and only $7 \%$ have 4 or more.

In 1987 there were 37 advertised positions in pediatric nephrology in the United States (4 in Canada) which remained unfilled. The projected need by chair program directors for additional pediatric nephrologists by 1992 is 75 in the United States (7 in Canada), for a total of 112 pediatric nephrologists for the United States (11 for Canada). Since the survey was completed in the summer/fall of 1987 , position changes have increased the projected manpower needs: at least 4 senior pediatric nephrologists will retire, and 4 more have or will leave pediatric nephrology for other responsibilities.

In 1985, 30 U.S. training programs in pediatric nephrology were accredited by the ACGME of the AMA. Moreover, the American Board of Pediatrics and its sub-board of Pediatric Nephrology changed the training required for sub-board eligibility from two to three years in an accredited program. Currently there are 46 trainees in 17 different accredited programs in the United States; however, only two-thirds of these trainees will be eligible to accept positions in the United States. During 1988, 1 pediatric nephrologist will complete a tour of military duty, and 7 others will complete training-the same number that are leaving pediatric nephrology by retirement or a change in responsibility. About 10 trainees per year will complete fellowships in 1989 and 1990. 


\section{GOALS TO IMPROVE THE QUALITY OF CARE FOR} CHILDREN WITH KIDNEY DISEASE

Following are a set of goals to improve the aims of the discipline of pediatric nephrology and thus to improve the quality of care.

1. Increased support for training pediatricians to become nephrologists and basic and clinical investigators in the area of pediatric nephrology.

2. Specific grant programs to support relevant basic and clinical research for pediatric nephrology.

3. Expanded support for multicenter clinical studies on the etiology and therapy of kidney disease in children.

4. Creation of two pediatric nephrology centers with a multiuniversity location.

I.A. Support of Training. In large part, pediatric nephrology sections are relatively small, usually containing no more than three faculty members, who carry a heavy clinical load. As noted previously, this has not necessarily precluded excellent clinical and basic research accomplishments with many pediatric renal programs. However, the small number of faculty in most sections of pediatric nephrology, combined with the clinical workload, has led to a necessity for sharply focused research, and many of these programs are disadvantaged when chairs or directors apply for training programs in pediatric nephrology, particularly when they are to be judged in competition with larger programs in adult nephrology or basic sciences.

It is imperative, therefore, that special consideration be given to the particularly unique circumstances which affect pediatric nephrology, but would not preclude the effective training of future clinical and basic investigators. It should be possible for a number of the accredited programs to demonstrate sufficient research activity to warrant the funding of fellowships so that future generations of pediatric nephrologists can attain specific credentials necessary for long-term academic careers. While the number of trainees and preceptors available in many of these programs would not seem to meet the "critical mass" that might be demonstrable in adult nephrology or basic science training programs, this could be overcome by a number of mechanisms so that the trainees in pediatric nephrology could be integrated into essential learning activities, such as journal club, structured core curricular teaching of scientific methodology and basic science aspects of nephrology, by inclusion in programs already in existence for the training of adult nephrology or basic science postdoctoral fellows. In addition, consideration can be given to the possibility of developing multi-university coordinated fellowship programs, where several universities associate in a conjoined program under one administrative core.

Current methods used to fund research fellowships in pediatric nephrology have inherent difficulties. The addition of a pediatric nephrology trainee to a currently existing training grant in adult nephrology requires a carefully coordinated effort between the directors of the pediatric and adult nephrology sections. This mechanism is not widely applicable because few university centers have a strong academic program in both disciplines and, in some instances, may require a pediatric nephrology applicant to devote major efforts to issues not of direct relevance to pediatric nephrology. Secondly, because of the particularly unique considerations discussed above, training grants awarded to individual pediatric nephrology sections are feasible in a very limited number of institutions. Pediatric nephrology trainees clearly need training in pediatric nephrology training programs as they will be expected to deal clinically and from a research perspective with the nephrologic disorders of childhood. While National Research Service Awards and Physician Scientist Awards are available and would meet the objectives of supporting pediatric nephrology training, the mechanism would not meet the manpower needs to insure the continued viability of research and academic productivity of the discipline. Fellowships from the National Kidney Foundation and the American Heart Association are also limited in number. Lastly, it is the expectation that local institutions would provide funds for training during the clinical year of the 3 year fellowship, but clinically derived or hospital-provided fellowship funds cannot be expected to support research training on an ongoing basis.

Consideration should be given to the development and funding of three or, preferably, four categorical pediatric nephrology training programs which could be administered and coordinated by a consortium of universities under an administrative core. Application for fellowship positions funded through the NIH, but administered by these universities would be evaluated by a fellowship training committee established by the nephrology divisions within these universities. Training would be funded by the traditional P-32 Training Grant. A pediatric nephrology training center would consist of pediatric nephrologists from three to five academic centers that could put together a consortium. Requirements from a center would include that faculty from the center be funded by the NIH or NSF and that a curriculum would be developed for trainees. The program would be exactly the same in the parent institutions. Special expertise in additional areas could be subcontracted out. The rotation could also be open to institutions without research support but strong in other areas. These institutions need not be limited to pediatrics and may include basic science and adult nephrology. The trainee would then rotate into those subcontracted institutions for a period of approximately 6 months. One could also envision three meetings a year of the program directors and trainees where the progress of research work could be discussed. Each network of universities would need to appoint a chair and a research training committee. This committee would provide for an ongoing assessment of the quality of education available to the fellows, a progressive review of their progress during fellowship, and an assessment of their productivity. A categorical fellowship program would allow the flexibility to insure the highest level of training in the maximum number of pediatric nephrology centers. It is intended that training programs would include formal courses in study design, biostatistics, and other course work in basic sciences that would be sufficient to begin to produce a capable and independent investigator. Finally, recognizing the modern revolution in biology, it is anticipated that fellows would be trained in such areas as cell biology, molecular biology, and cell culture techniques and that their studies have a strong biochemical emphasis, so that they can approach the major problems of pediatric nephrology with an appropriate degree of scientific sophistication. In some centers only one fellow would be funded until the completion of his or her program, while in others a position could be funded annually, if justified by the scope of the program. Thus, based on the manpower needs indicated previously, the NIH could fund 10 to 12 fellowship positions per year to be administered by this mechanism. This would not obligate long-term funding for any one program but would allow successful programs to compete on a continuing basis. Fellowship positions funded by this mechanism would be applicable for two years of research training, but not for training in clinical nephrology.

A proposed mechanism to fund several categorical pediatric nephrology training programs to meet these needs would be the commitment of 0.25 to $0.5 \%$ of current training funds from appropriate NIH institutes which presently fund research in renal disease. From a broad spectrum of research activities, appropriate institutes include NIDDK, NIHLB, NIAID, GM, NIAMSD, and NICHD. The responsibility for development and oversight of this program would fall to the NIDDK or NICHD, because the latter institutes have a concern for scientific investigation in renal development and disease and the long term well being of children. This multiuniversity categorical pediatric nephrology program would meet the projected need for new pediatric nephrology investigators at between 10 and 12 trainees per year. 
Faculty retention. The overall manpower needs cannot be met if there continues to be significant attrition of pediatric nephrologists. Therefore, consideration should be given to the development of a proposal which would provide funds for pediatric nephrologists on academic faculties to redefine and develop their investigative skills. Over the past 15 years, a number of individuals have assumed the role as director of a section of pediatric nephrology immediately upon emerging from their fellowship training. In most instances, the need for a section of pediatric nephrology was dependent upon clinical needs which were not being met. Therefore, these individuals were frequently faced with a large population of patients with chronic renal problems and were unable to continue to develop their research and academic skills. As some of these programs have been able to add a second or third pediatric nephrologist, it has been clear that there is a need for individuals to have the opportunity to reimmerse themselves in basic and/or clinical investigation. However, it is extremely difficult for such individuals to generate funding for such retraining.

A second group of individuals who might be eligible for such an award would be former trainees who spent only two years in a pediatric nephrology program and, therefore, did not have an opportunity to completely develop their investigative talents. While the American Board of Pediatrics' Sub-board of Nephrology now recognizes the need for trainees to undergo at least 3 years of training in an approved pediatric nephrology training program, this requirement becomes effective in 1988. For individuals with only 2 years of fellowship training, it is difficult to be competitive for continued funding of their research efforts, and they need to have a period of time in which they can continue to develop their investigative abilities. While a number of colleagues and collaborators are usually willing to provide the intellectual support necessary to allow such individuals to develop, it is rare that adequate funding is available to support these types of investigative efforts. Special consideration for pediatric nephrologists should be given in the utilization of existing funding mechanisms.

I.B. Specific training recommendations. A series of specific recommendations with regard to training follows.

1. Recognize the need to categorically support pediatric nephrology research trainees with ongoing and new training mechanisms.

2. Allow flexibility in the development of training programs.

a. Large programs with a critical mass could submit institutional nephrology training program proposals.

b. At least three or four categorical training programs should be developed with a multi-university consortium functioning as a clearing house. This consortium would need to remain impartial in the granting of trainee slots to applicant divisions of pediatric nephrology. This consortium would also advertise the availability of such training positions to pediatric departments. At least 10 to 12 positions would be available annually.

3. Recognize the need for sabbatical training, either a Senior International Fellowship (Fogarty) (F06) or a Senior Scientist Research Award (F35) to foster mid-career changes in direction of research. While this mechanism is in place within the NIDDK, its use should be encouraged. As with trainees, these faculty members applying for mid-career re-education grants should be encouraged to emphasize basic science during their laboratory experience, lasting perhaps 12 months, so that they can use these newly gained skills to answer questions of relevance to the field of pediatric nephrology.

II.A. Development of grant programs or RFA of relevance to pediatric nephrology. In addition to greater support of training for pediatric nephrology, there is a need to encourage the NIH to support special areas of research having relevance for the discipline of pediatric nephrology. At least four areas of research activity require specialized support: the biology of maturation and differentiation of the renal system, including congenital renal disorders; neonatal factors in health and disease that have ramifications for later life; study of disorders which have antecedents in childhood but are traditionally expressed in adult life; and study of problems involving infants and young children with renal insufficiency, including growth, nutrition, renal osteodystrophy, hypertension, dialysis, and transplantation.

The biology of maturation and differentiation of the renal system is an area of research activity concerned with questions regarding important frontier areas of developmental renal physiology and biochemistry. These areas include ontogeny of the renal vasculature, the glomerulus (both its composition and function), various nephron segments, and the lower urinary tract. Hypothesis testing involves the use of micropuncture, microperfusion, isolated glomerular preparations, cell and fetal organ culture, cell membrane separation, epithelial cell techniques (toad and turtle bladder), in vivo cannulation of fetal animals (sheep), receptor biochemistry, cell membrane biochemistry, regulation of gene expression (cDNA probes), cellular and humoral immunologic methodologies, and whole animal studies (growth). Among the general questions to be asked is how the developing kidney can support the endocrinologic, metabolic, nutritional, excretory, and body composition and acid-base regulation of the growing organism.

Such studies need to be examined within the context of the regulation of the system of function to be examined. Not only is there some need to have a better description of the ontogeny of transport and metabolic pathways, but virtually nothing is known concerning the intracellular events underlying the differences between developing and mature systems in transport or secretory capacity of the developing nephron. The techniques of isolation of transporters, their sequencing and cloning has not been applied to the developing organism. Because of differences in hormone receptor density and/or function and signal transduction, a better appreciation of the integrative physiologic responses and interaction of hormones is needed. Study of the biology of maturation and differentiation should involve a more complete understanding of the nature of renal hypertrophy in the young. Finally, the paucity of glomerular disorders and the virtual lack of immunemediated disorders during infancy point out that the neonatal kidney may respond differently to immunologic mechanisms that cause glomerular and tubular interstitial nephritis in older children and adults. These areas of investigation have the potential to identify not only the chronology of maturational events, but basic cellular/molecular mechanisms as they affect the more complex process at maturity.

With the ever-increasing improvement in neonatal mortality and in the care of very small preterm infants (particularly those under $1000 \mathrm{~g}$ birth weight), neonates enter the world before renal morphogenesis is complete. Many of these infants are ill, require intricate treatments both in terms of immediate resuscitation and chronic care and feeding, and are exposed to powerful pharmacologic agents, nutrient solutions, radiologic agents and infectious agents. These factors may alter, impair or enhance renal, genitourinary and renal vascular development and may have ramifications for later life. Examples include the propensity to develop renal ischemia or infarcts secondary to indwelling umbilical arterial catheters, which lead to renal artery thrombi and severe hypertension; the development of nephrolithiasis in relation to the use of loop diuretics for bronchopulmonary dysplasia, parenteral nutrition and low phosphate infant formulas that lead to rickets and hypercalcuria; the propensity for infection and urinary reflux as a consequence of indwelling urinary catheters or bacteremic episodes; and the use of drugs which result in hyperexcretion of various ions or organic solutes despite a greater need for these substances in the growing child. The developing kidney is unique in that epithelial growth factor is low, renal blood flow is low, and sodium, potassium, phosphate, bicarbonate, and organic solute excretion differ from the mature kidney. Mesangial cell development is incomplete, and 
the function of receptors influencing glomerular hemodynamics in terms of adrenergic and dopaminergic stimuli are incompletely activated. Receptors for other hormones, including parathyroid hormone, antidiuretic hormone, and EGF, prostaglandins and the renin-angiotensin-aldosterone axis also are maturing. Investigations in this area are important in the short term for the direct clinical management of these infants, and studies of these parameters may identify risk factors with long-term ramifications.

Diseases which result in chronic renal failure in adults, such as polycystic kidney disease, Alport's syndrome, diabetes mellitus, cystinuria, reflux nephropathy, systemic lupus erythematosus, frequently have their onset during childhood. Thus, if these diseases can be prevented or modified at the time of their onset, the financial and emotional burdens of later life will be alleviated. It is the responsibility of the pediatrician and the pediatric nephrologist to investigate ways to treat infants and children to modify a pattern that will have disastrous results in adulthood.

With the almost universal application of the techniques of dialysis and transplantation to infants and young children with renal insufficiency and the use of modern therapeutic strategies to forestall growth failure, osteodystrophy, and systemic metabolic acidosis, the need exists both to develop a data base and to ask questions of clinical importance. Little is known regarding the role of in utero intervention in preventing the damage caused during the first and second trimester of pregnancy to the developing fetus. The long term effects of toxin-induced or hypoxiarelated acute renal failure in the pre-term or term neonate are also uncertain. Factors such as the appropriateness of nutritional supplements, their protein and lipid composition, the appropriate dose and route of administration of vitamin D metabolites, the need to restrict phosphate intake, and the social and psychological needs of the infant and his/her parents are research areas worthy of more extensive investigation. Additional questions can be raised regarding the role of uremia on protein synthesis and amino acid utilization in young infants, the apparent failure of growth hormone to elicit somatomedin formation and action, and the loss of concentrating capacity of the remaining nephrons. The progression to renal insufficiency, despite appropriate correction of congenital urinary tract obstruction, remains an enigma. Fundamental issues, such as the timing of dialysis treatment, the need for early transplantation, and the effects of these therapies on stature and motor and central nervous system development are not clear. Experimental issues can involve the use of animal models in some instances, but multicenter clinical studies are indicated to answer many of these questions. Finally, tissue culture of the fetal kidney from animals demonstrating in utero obstruction may provide a method to study calcitriol synthesis, renal acidification mechanisms, and hypertrophy/hyperplasia-related issues.

II.B. Specific research support recommendations.

1. Four broad categories of research activities of high relevance to central issues in pediatric nephrology have been identified.

2. Key personnel at various funding agencies and especially at the NIH need to be made aware of research needs in these areas for the potential development of RFA.

3. Appropriate mechanisms for the review of proposals directed toward these research questions are warranted.

a. Increase by $10 \%$ the number of pediatric nephrologists on study sections and initial review groups to which such proposals would be submitted.

b. Consider an Ad Hoc Study Section on an annual basis composed of pediatric nephrologists sitting on other Study Sections or who have served previously on NIH Study Sections.

c. Constitute a Special Study Section dedicated to reviewing applications in response to RFA directed toward these questions.
III.A. Multicenter clinical studies. The pediatric nephrology community has successfully developed several multicenter study groups, including the International Study of Kidney Disease in Childhood (ISKDC) to study glomerular disease in childhood; the Southwest Pediatric Nephrology Study Group (SPNSG) to study glomerular disease, hematuria/hypercalcuria, nutrition, and vesicoureteric reflux; the Growth Failure in Children with Renal Disease Study Group (GFRD) to examine growth in uremic children in relation to renal osteodystrophy; the Renal Transplant Data Collection System to examine factors relating to the success of renal transplants; and a multicenter trial of high dose recombinant growth hormone in children to determine its influence on growth. These organizations and multicenter study groups are evidence of the spirit of cooperation within the community of pediatric nephrology.

At present the Chronic Renal Disease Program of the NIDDK is funding multicenter clinical studies as identified previously. One examines growth in older children with chronic renal failure (GFRD), and the other examines the influence of protein intake on measurement of the progression of renal failure in infants with chronic renal insufficiency. These involve investigators from 19 medical centers, the use of intricate data collection and analysis, the mailing of patient samples to a centralized laboratory facility, detailed analysis of caloric, water, and diet composition of enrolled patients, and an independent medical and statistical advisory board capable of detecting flaws in study design.

The basis of clinical research is the double blind controlled study, by which therapeutic approaches can be critically evaluated. Because of the limited number of patients at any center, a multicenter approach offers the only feasible alternative. Further, the multicenter clinical study can employ the appropriate statistical input and laboratory controls necessary to develop and analyze meaningful data in order to reach rational conclusions.

Existing multicenter consortiums should be encouraged to undertake clinical research projects related to questions posed in Section II. As a cost-effective method, new centers could be included in existing networks. Many of the problems relating to the consequences of neonatal renal injury, to the recognition that adult disease have their antecedents in childhood, and to the effects of renal disease on the young, rapidly growing child can be examined using carefully designed studies. With the advent of impressive technologies in immunogenetics and restrictionlinked fragment polymorphism analysis, multiple cooperatives can be used to examine genetic disorders in a much larger population base. It would be prudent to initiate new research protocols using currently existing multicenter study groups.

III.B. Specific recommendations for multicenter trials.

\section{Continued support for multicenter trials is desired.}

2. Existing core structures could be adapted to answer newly posed questions.

3. The ASPN should develop a Research Committee to screen potential research protocols and assess their suitability.

IV.A. Development of two pediatric nephrology centers. With the recent establishment of kidney and urology centers having focused areas of research, the goal of developing centers emphasizing pediatric nephrologic disorders should receive serious consideration. Each center could be focused around pediatric nephrologic problems and located in a given geographic region, thereby encompassing the faculty at several university medical centers. Appropriate topics for center activities might include biology of renal development; prevention of renal disease during childhood; and immunologic renal disorders affecting children.

A center could encompass the facilities of 7 to 10 universities in a region with a central administrative office and separate funding for professional and technical personnel to each university in the consortium. Equipment and supplies could also be assigned in accordance with a given protocol. These pediatric kidney centers would serve as a means of improving the status of pediatric nephrology. 


\section{CONCLUSION}

This position paper recognized the rather tenuous state of the discipline of pediatric nephrology and the currently limited entry of trainees into the discipline. At least three major recommendations are made to insure its future. First, a flexible system of providing training opportunities for postdoctoral fellows and mid-career academicians is recommended. Second, emphasis should be placed on four areas of research which are of clinical and scientific relevance to pediatric nephrology. Third, the expanded use of multicenter study groups will form a more effective means of answering important clinical questions. This prescription will expand the data base in the discipline and more completely develop our understanding of the biology of the maturing kidney. It has the potential to lead to more useful therapeutic approaches while assuring a pool of capably trained academicians who can better function within the mission of an academic medical center and who will have the expertise to conduct independent research.

Writing committee: Russell W. Chesney, Coordinator; Billy S. Arant, Jr.; Gladys Hirschmann; Pedro A. Jose; Antonia C. Novello; Norman J. Siegel

ASPN council: Ira Greifer, Barbara R. Cole, Robert L. Chevalier, Raymond D. Adelman, Jean Robillard

American Board of Pediatrics: Robert Ettenger, Julie Inglefinger, John Hoyer, Robert McLean, F. Bruder Stapleton

Consultants: John Lewy, Malcolm Holliday, Robert Vernier, Paul McEnery, Richard Fine, Chester M. Edelmann, Jr;; Adrian Spitzer, Warren Grupe; Ellis D. Avner; James C.M. Chan; Alan Gruskin; Michael Bailie 